\title{
Transposon Screen of Surface Accessibility in S.
}

\section{aureus}

Noel J. Ferraro and Marcos M. Pires ${ }^{\star}$

${ }^{1}$ Department of Chemistry

University of Virginia

Charlottesville, VA 22904, USA

*corresponding author: mpires@virginia.edu 


\section{Abstract}

Bacterial cell walls represent one of the most prominent targets of antibacterial agents. These agents include natural products (e.g., vancomycin) and proteins stemming from the innate immune system (e.g., peptidoglycan-recognition proteins and lysostaphin). Among bacterial pathogens that infect humans, Staphylococcus aureus (S. aureus) continues to impose a tremendous healthcare burden across the globe. $S$. aureus has evolved countermeasures that can directly restrict the accessibility of innate immune proteins, effectively protecting itself from threats that target key cell well components. We recently described a novel assay that directly reports on the accessibility of molecules to the peptidoglycan layer within the bacterial cell wall of $S$. aureus. The assay relies on sitespecific chemical remodeling of the peptidoglycan with a biorthogonal handle. Here, we disclose the application of our assay to a screen of a nonredundant transposon mutant library for susceptibility of the peptidoglycan layer with the goal of identifying genes that contribute to the control of cell surface accessibility. We discovered several genes that resulted in higher accessibility levels to the peptidoglycan layer and showed that these genes modulate sensitivity to lysostaphin. These results indicate that this assay platform can be leveraged to gain further insight into the biology of bacterial cell surfaces. 


\section{Introduction}

Bacterial resistance to antibiotics has become an imminent threat to global health and must be met with an antibiotic pipeline revitalization or, in lieu of that, alternative methods to combat bacterial infections. Intrinsic resistance is a multifactorial phenomenon but sometimes it can be mediated by a simple lack of accessibility to the bacterial targets. ${ }^{1-3}$ Recent efforts have led to the discovery of molecules that can potentiate antibiotics by improving permeation to and across the bacterial cell surface. For example, Gramnegative pathogens can be sensitized by co-treatment with polymyxins (and similar outer membrane destabilizers), which improve permeation of antimicrobials through the bacterial cell wall. ${ }^{4-8}$ Likewise, positively charged Branched PolyEthylenImine (BPEI) has been shown to potentiate $\beta$-lactam antibiotics against the Gram-positive pathogen methicillin resistant Staphylococcus aureus (MRSA). ${ }^{9}, 10$ It was proposed that BPEI neutralization of negatively charged polymers on the bacterial cell surface improved permeability. ${ }^{11}$ These examples demonstrate that improved permeation to the essential cell wall components can be a powerful modality of reducing intrinsic resistance to small molecule antibacterials and immune proteins. ${ }^{12}$

The cell walls of bacteria are complex in structure and composition. For the Gram-positive bacterium, Staphylococcus aureus (S. aureus), the cell wall is composed of a thick peptidoglycan (PG) scaffold that is shielded by proteins and other biomacromolecules (Figure 1). ${ }^{13}$ The proteins are covalently anchored onto the PG via the transpeptidase, sortase A. ${ }^{14,15}$ The cell surface is further functionalized by wall teichoic acids (WTAs), 
which are anionic glycopolymers that are covalently anchored onto the PG, and lipoteichoic acids (membrane anchored). ${ }^{16}$ The restricted accessibility afforded by teichoic acids and other surface-bound macromolecules has been implicated in the virulence of Staphylococci. In Drosophila, it was recently discovered that the accessibility of peptidoglycan recognition proteins (PGRPs) to PG plays a determinant role in the host immunity to infection. ${ }^{17}$ Removal of WTAs resulted in considerably increased accessibility of PGRPs to the PG and predisposed S. aureus to PGRP-mediated immunity. Additionally, it has been found that WTA-deficient S. aureus fails to colonize the nasal cavities of rats. ${ }^{18}$ More recently, it was established that Staphylococcus epidermidis shift from a commensal to pathogen lifestyle upon expression of S. aureus-like WTAs. ${ }^{19}$ Aside from modulating immunity, PG accessibility can potentially impact drug discovery based on the fact that a number of FDA approved antibiotics work by inhibiting steps in the bacterial PG biosynthesis pathway. ${ }^{20,21}$ The essential nature of accessibility to the PG scaffold by immune proteins and antibiotics alike means that there is a need to better understand the genes that control surface accessibility. 


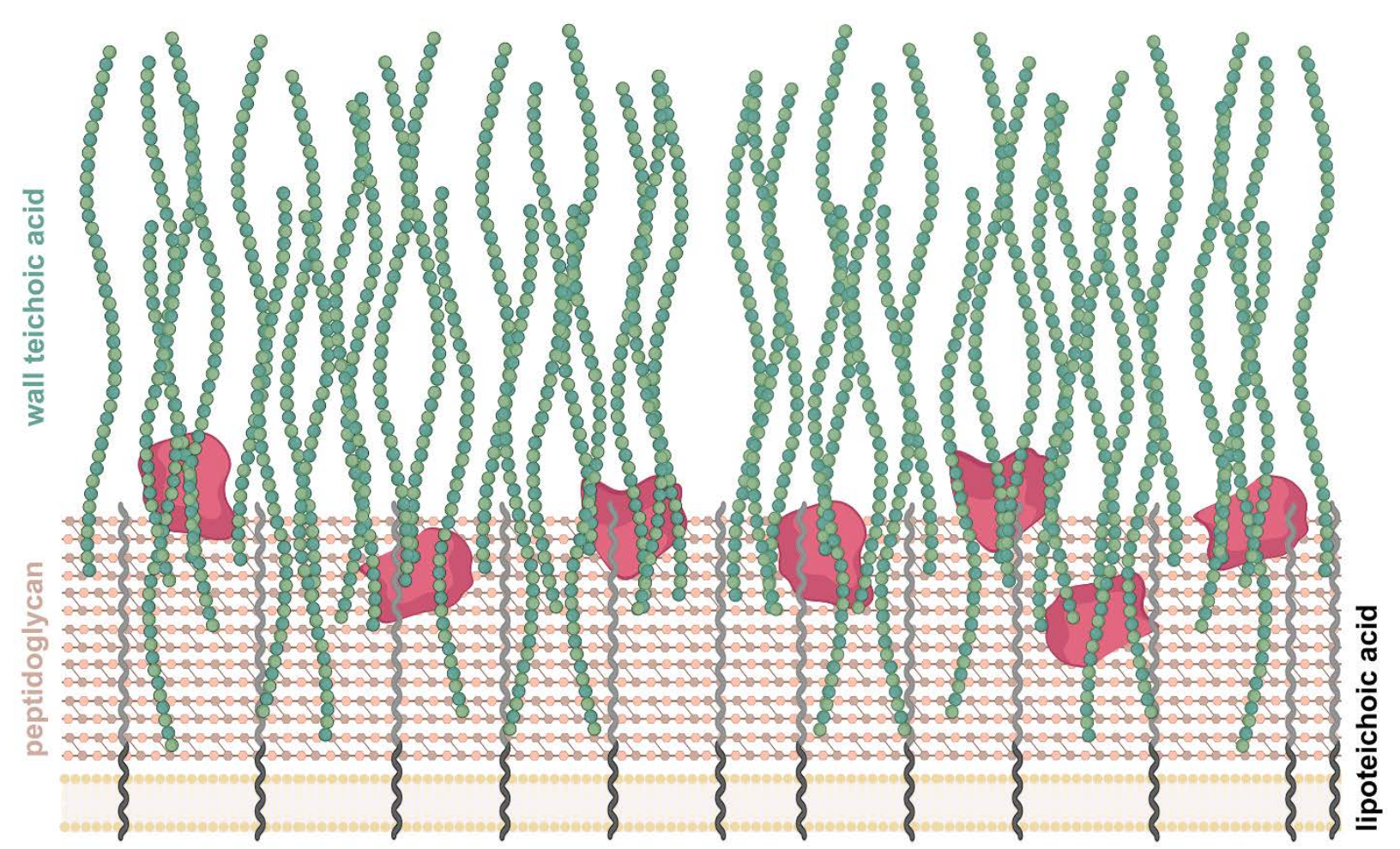

Figure 1. Schematic representation of the surface of Gram-positive bacteria. The PG scaffold is heavily decorated with a number of biomacromolecules, including WTAs, LTAs, and proteins. These features can reduce the permeation of extracellular proteins and molecules to the PG scaffold, which can modulate host immunity and the potency of antibacterial agents.

Among Gram-positive pathogens, S. aureus has proven to be particularly challenging to treat; it is a formidable foe, as it is well suited to evade attacks by the host immune system $^{22}$ and it is a pathogen that can readily become resistant to standard of care therapies. ${ }^{23}$ The difficulty in finding new efficacious antibiotics against $S$. aureus highlights the need to explore less conventional therapeutic approaches such as antibiotic adjuvants or immunotherapies. ${ }^{24-29}$ For example, adjuvants can potentiate antibiotics by improving 
their accessibility to their cognate molecular targets. Likewise, anti-infective immunotherapeutics (e.g., antibody recruiting agents developed by our lab $24-27,29$ ) work by targeting specific macromolecules on bacterial cell surfaces. Despite the pivotal role of surface accessibility in bacterial pathogenesis, to date there has not been a systematic analysis of the genes that control penetration of biomacromolecules across cell surfaces of S. aureus; information that can directly impact current therapies and the development of alternative treatments. To address this, we performed a screen against a nonredundant transposon mutant library using a robust assay that reports on surface accessibility in S. aureus.

\section{Results and Discussion}

We set out to comprehensively screen nonessential genes in S. aureus for their ability to alter accessibility to the PG scaffold. The basis of the screening assay is site-specific incorporation of a biorthogonal handle via metabolic remodeling of bacterial PG. ${ }^{11}$ More specifically, an unnatural amino acid (D-LysAz) is supplemented in the media, inoculated with S. aureus, and cultured overnight (Figure 2A). During PG biosynthesis and assembly, transpeptidases catalyze the replacement of the $5^{\text {th }}$ position D-alanine on the stem peptide within the bacterial PG scaffold for D-LysAz, thus leading to the covalent installation of the azido-handle (Figure 2B). With the azido group installed through the entire PG scaffold, treatment of cells with a complementary DiBenzoCycloOctyne (DBCO) handle conjugated to fluorescein (DBCOfl) leads to the specific tagging of the bacterial PG with fluorescent moieties (Figure 2C). 30,31 We previously showed that cellular fluorescence levels reflect the ability of molecules to reach the PG scaffold and 
consequently result in a covalent tag. We hypothesized that this reporter assay could be leveraged to identify genes that modulate PG accessibility in S. aureus, thus revealing genes that have implications in immunity and drug discovery. By screening a nonredundant library of transposon mutants, we reasoned that any mutants exhibiting increased cellular fluorescence levels could identify potential genetic alterations that result in higher surface accessibility.

A

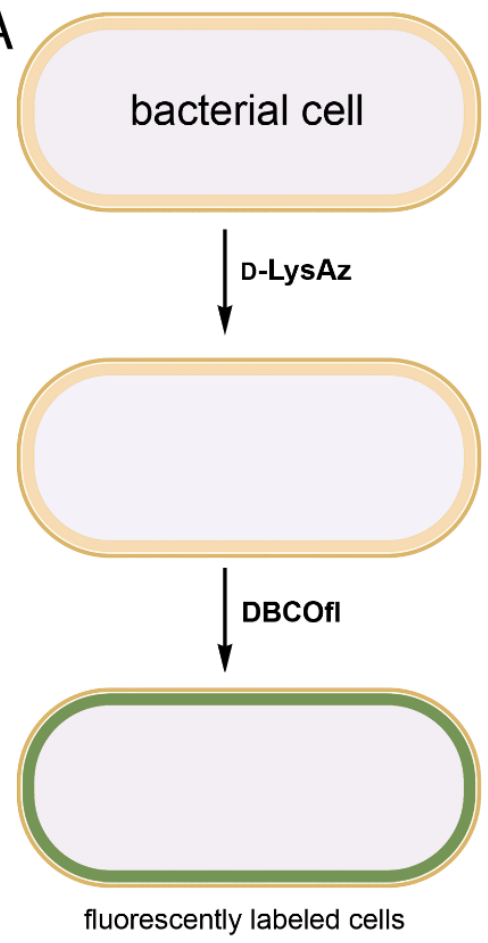

B

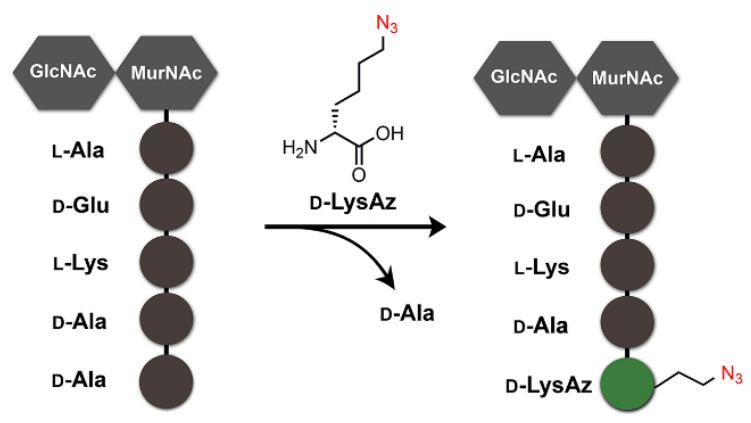

C

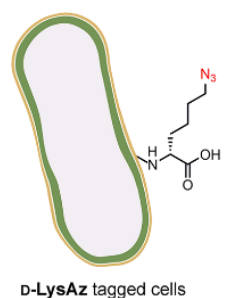

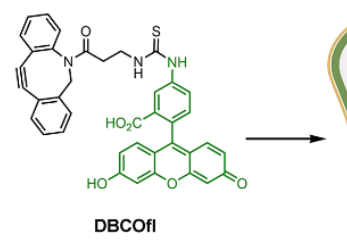

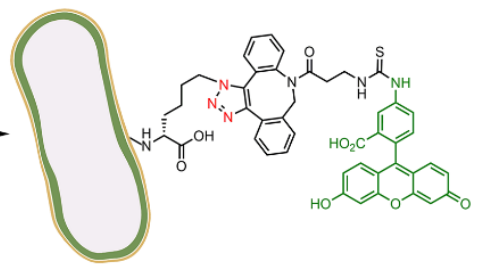

Figure 2. (A) Live cell labeling with D-LysAz and DBCOfl. Cells are analyzed by the flow cytometer to quantify modification with DBCOfI. (B) The unnatural amino acid (D-LysAz) is enzymatically installed in place of the terminal D-alanine on the stem peptide within the bacterial PG during cellular growth. (C) Chemical structures depicting the reaction between the azido moiety and DBCO. 
First, we demonstrated that the assay preformed as expected in wildtype (WT) S. aureus cells by supplementing the PG label (D-LysAz) during overnight culture. Subsequent treatment with DBCOfl resulted in cellular fluorescence levels that were $\sim 6$-fold and $\sim 8$ fold higher than no amino acid and the L-LysAz, respectively (Figure 3A). We $\mathrm{W}^{32}$, 33, and others $^{34-37}$, had previously demonstrated that the enantiomeric L-LysAz does not incorporate into the growing PG scaffold of bacteria. These results confirm that cellular fluorescence increases in a manner that is dependent on PG remodeling with azido handles. To demonstrate the effect of surface accessibility, a similar labeling protocol was performed with tarO-deleted S. aureus, which results in WTA-free S. aureus cells. ${ }^{16,} 38,39$ Fluorescence levels of $\Delta \operatorname{tarO}$ S. aureus labeled with D-LysAz were $\sim 2$-fold higher than WT cells (Figure 3A). These results are consistent with previous findings showing that WTAs control the accessibility of immunoproteins to the PG. ${ }^{17,40,41}$

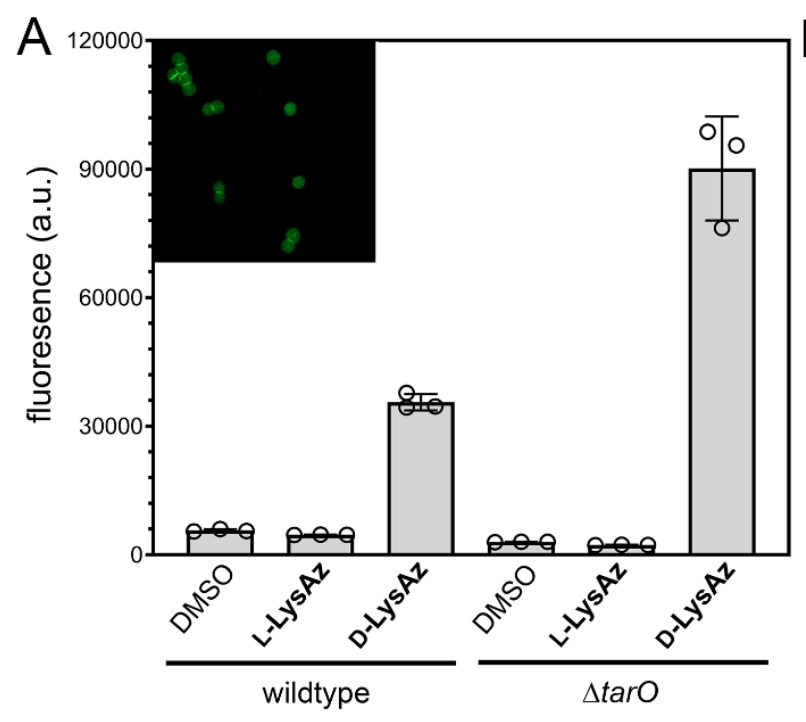

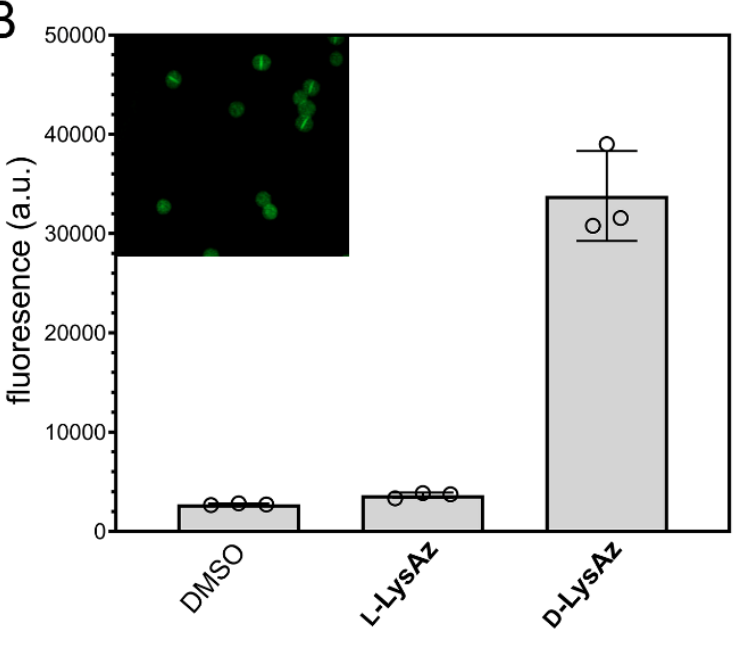


Figure 3. (A) Flow cytometry analysis of WT S. aureus (ATCC 25923) or S. aureus ( $\Delta$ tarO) treated overnight with DMSO, 1 mM of L-LysAz, or 1 mM of D-LysAz followed by a treatment with $25 \mu \mathrm{M}$ of DBCO-FI. Inset, confocal microscopy of the WT cells tagged with and treated with D-LysAz followed by a treatment with $25 \mu \mathrm{M}$ of DBCOfI. (B) Sacculi isolated from WT S. aureus (ATCC 25923). WT cells were incubated with 1 mM of DLysAz, 1 mM of L-LysAz, or DMSO alone overnight. Next, the cells were treated with 25 $\mu \mathrm{M}$ of DBCO-FI and subjected to a sacculi isolation protocol. The resulting sacculi were analyzed by flow cytometry. Inset, confocal microscopy of the sacculi from WT cells that were treated with D-LysAz followed by a treatment with $25 \mu \mathrm{M}$ of DBCOfI. Data are represented as mean $+/-\operatorname{SD}(n=3)$.

We then performed an additional set of experiments to confirm that the fluorescent reporter handle reached the PG scaffold. Our research lab recently described an assay (SaccuFlow) that combines the quantification of flow cytometry with the analysis of bacterial sacculi. ${ }^{11}$ Similar to the whole cell assay described in Figure 2, S. aureus cells were treated with D-LysAz followed by DBCOfl. Instead of analyzing the whole cell, the sacculus was isolated using a standard isolation procedure which includes boiling of cells with SDS (to solubilize biomacromolecules) and treatment with trypsin (to remove proteins anchored on the PG scaffold). Following these steps, the isolated sacculi are analyzed on the flow cytometer. As expected, fluorescence levels of sacculi from cells treated with a combination of D-LysAz and DBCOfI were $\sim 12$-fold and $\sim 9$-fold higher than sacculi from cells treated with DMSO or L-LysAz, respectively (Figure 3B). Confocal microscopy confirmed that the whole cells and the isolated sacculi retained the expected 
size and shape of the fluorescently labeled cells and biomacromolecule, respectively (insets of Figure 3). To confirm the metabolic labeling step did not alter cellular viability, a growth curve analysis was performed by monitoring the optical density at $600 \mathrm{~nm}$ (Figure S1). Consistent with our prior results using D-amino acid labeling at the concentrations used in our assay, cellular viability was not impacted. Together, these results show that the combination of PG labeling with D-Lys Az and treatment with the DBCOfI in the media results in the fluorescence tagging of bacterial PG in a manner that reports on cell surface accessibility.

We next shifted our efforts to demonstrate the utility of the surface accessibility assay by screening a library of $S$. aureus transposon mutants to identify genes that could play important roles in controlling permeation to the $S$. aureus cell surface. To this end, a transposon $(T n)$ insertion mutant library was used as the platform of the screen, which contained 1952 individual strains each with a single insertion within a nonessential gene in S. aureus USA300. A major advantage of using the transposon mutant library is the potential to identify genes that were not previously purported to have biological roles related to accessibility, thus revealing new targets for the development of adjuvant agents. Before screening the entire library, the assay was benchmarked in a 384-well format and it was found that the assay could be readily miniaturized (Figure S2). Next, the entire library was screened using the combination of D-LysAz and DBCOfI (Figure 4A). While most transposon mutants did not significantly display altered labeling levels, several transposants exhibited elevated fluorescence levels (defined as 33\%+ above the 
average and denoted by the green line). These results suggest that those genes may potentially play a role in surface accessibility.

The 24 transposants with the highest increase in cellular fluorescence were re-assayed in triplicate and transposon mutants that consistently labeled at a higher level than wildtype were selected (Figure S3). These results showed that four transposants were confirmed to play a determinant role in the accessibility of DBCOfI to the cell surface, namely SAUSA300_1989, SAUSA300_1984, SAUSA300_1992, and SAUSA300_1467. SAUSA300_1989 and SAUSA300_1992 have been annotated as agrB and agrA, respectively. ${ }^{42}$ Their protein products play a central role in processing autoinducing peptides in S. aureus. Relatedly, the protein product of SAUSA300_1984 (mroQ) acts within the Agr pathway. ${ }^{43}$ Moreover, the protein product of SAUSA300_1467 (IpdA) has been shown to have a role in branched-chain fatty acid biosynthesis. ${ }^{44}$ The selected transposants were also tested for labeling with a second fluorescent probe (DBCOAF488) in order to determine the role of the fluorophore in PG labeling (Figure S4). The labeling pattern of the DBCO-AF488 mirrored that of DBCOfI with the mutants labeling at a higher level than WT. Similarly, we evaluated the impact of a spacer length between the DBCO handle and the fluorescein moiety across the transposants. A polyethylene glycol spacer was inserted between DBCO and fluorescein (DBCOpegfl) to test how a larger molecule would compare to the smaller DBCOfI. It was observed that increasing the size of the probe resulted in similar profile to that of DBCOfI, which indicates that the effect of increased fluorescence in the transposants as compared to WT is relevant for different sized probes (Figure S5). 
Finally, the identified hits were tested for their susceptibility to a larger molecular weight protein, lysostaphin, which needs to reach the PG layer to impart its antimicrobial activity (Figure 4B). We reasoned that genes that impact surface accessibility may be able to modulate the activity of lysostaphin by increasing its access to its target biomacromolecule, the bacterial PG. Lysostaphin is a bacteriocin that cleaves the pentaglycine cross-bridges found in the cell walls of S. aureus, leading to bacterial lysis. ${ }^{45}$ Challenge with lysostaphin showed that disruption to those genes resulted in altered sensitivity, which suggests the possibility that surface accessibility is playing a role lysostaphin activity. Together, this pilot screen confirmed the ability of the assay to be miniaturized to formats that are compatible with high-throughput screening and it reveals potential genes that may control surface accessibility of $S$. aureus. 

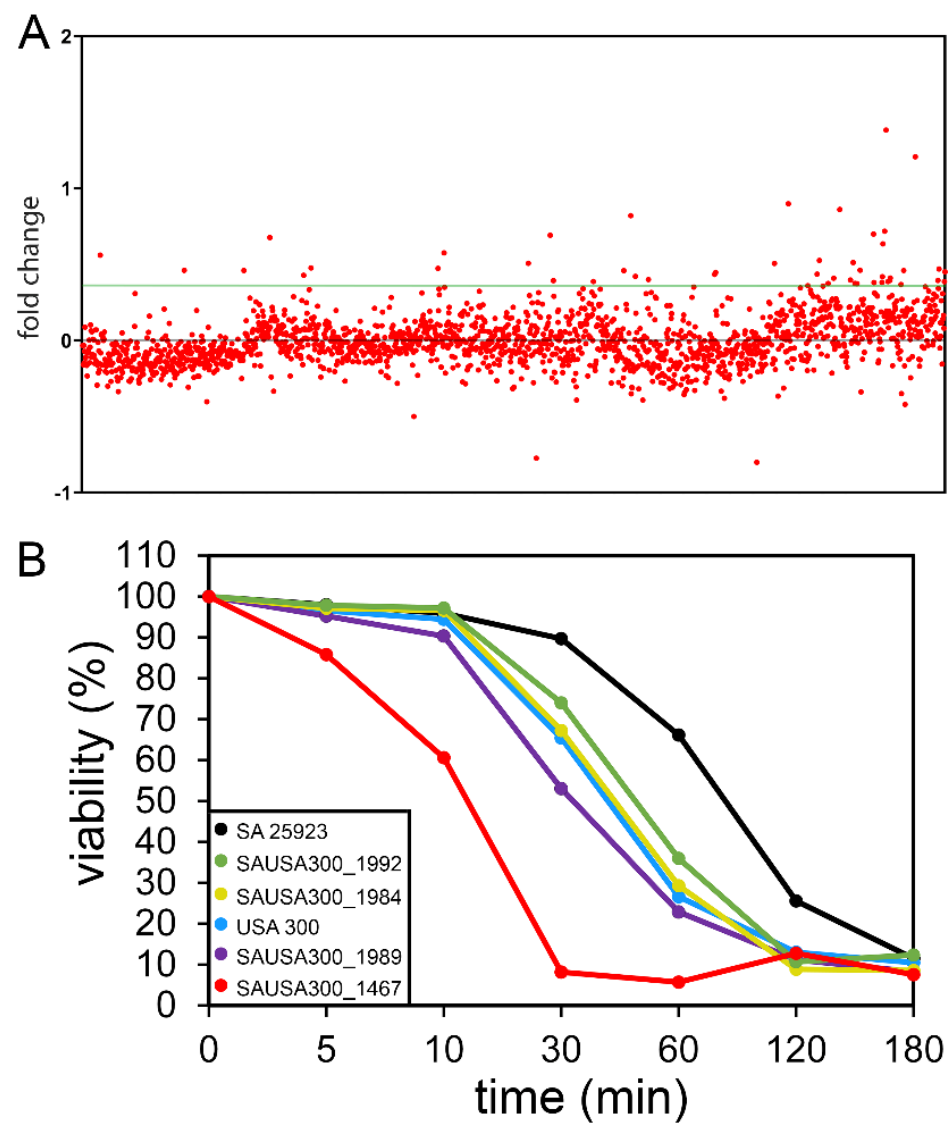

Figure 4. (A) Flow cytometry analysis of transposon library in S. aureus (ATCC 25923) treated overnight with $1 \mathrm{mM}$ of D-LysAz, followed by a treatment with $25 \mu \mathrm{M}$ of DBCOfl. Fluorescence levels were normalized to the average levels across all transposon mutants. Fold change refers to the ratio of fluorescence of the strain relative to the library average. Black line represents the zero point (average) and the green line represents +0.33 -fold change above the average, which we designated as potential hits. (B) Percent viability of designated transposon mutants and wild-type strains of $S$. aureus when challenged with $5 \mu \mathrm{g} / \mathrm{mL}$ of lysostaphin. Measurement of cellular viability was performed by analyzing the optical density at $600 \mathrm{~nm}$ and at each time point the optical density value was compared to the initial optical density reading. 


\section{Conclusion}

In conclusion, we have shown the application of our novel assay that reports on accessibility to the PG in identifying genes that potentially control permeation to the bacterial cell surface in S. aureus. Using site-selective incorporation of a biorthogonal handle and a corresponding reactive fluorescent probe, we were able to screen a transposon mutant library in effort to discern any genetic variations that led to an increase in fluorescence labeling thus signaling a possible increase in permeability. The screen revealed four transposants that consistently labeled at a higher level when compared to WT cells and some of these identified hits showed increased susceptibility to the bacteriocin, lysostaphin. The protein products of these transposants can potentially be considered new targets to develop potentiators for antibacterial therapies. Overall, we have demonstrated the use of our PG accessibility assay in a high-throughput screen and identified genes that may control access to the cell wall surface in S. aureus. 


\section{Table of Contents Figure}

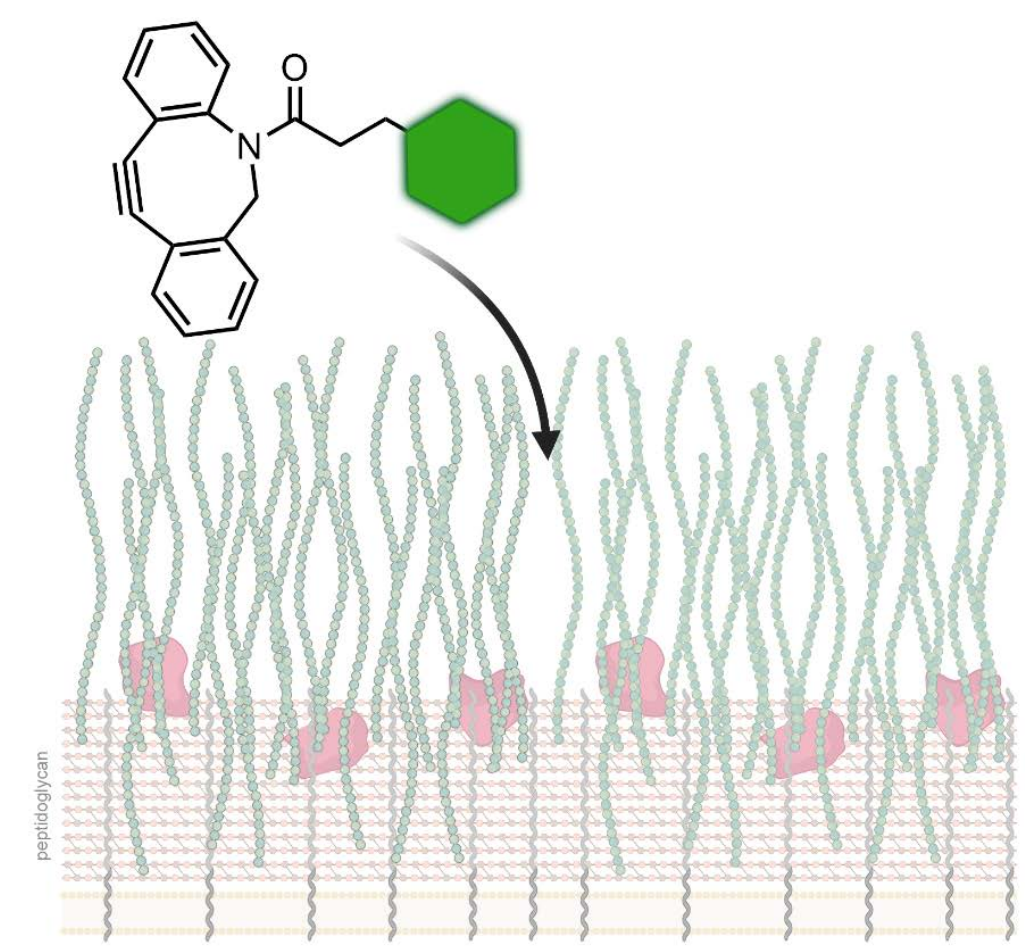

wildtype strain $=$ baseline fluorescence

$$
\begin{aligned}
& \text { screen of 1900+ transposon library in } \\
& \text { S. aureus for transposants with higher } \\
& \text { fluorescence levels (higher accessibility) }
\end{aligned}
$$




\section{References}

1. Cox, G., and Wright, G. D. (2013) Intrinsic antibiotic resistance: mechanisms, origins, challenges and solutions, Int J Med Microbiol 303, 287-292.

2. Martinez, J. L. (2014) General principles of antibiotic resistance in bacteria, Drug Discov Today Technol 11, 33-39.

3. Nuri, R., Shprung, T., and Shai, Y. (2015) Defensive remodeling: How bacterial surface properties and biofilm formation promote resistance to antimicrobial peptides, Biochim Biophys Acta 1848, 3089-3100.

4. French, S., Farha, M., Ellis, M. J., Sameer, Z., Cote, J. P., Cotroneo, N., Lister, T., Rubio, A., and Brown, E. D. (2020) Potentiation of Antibiotics against GramNegative Bacteria by Polymyxin B Analogue SPR741 from Unique Perturbation of the Outer Membrane, ACS Infect Dis 6, 1405-1412.

5. Vaara, M., and Porro, M. (1996) Group of peptides that act synergistically with hydrophobic antibiotics against gram-negative enteric bacteria, Antimicrob Agents Chemother 40, 1801-1805.

6. Klobucar, K., Cote, J. P., French, S., Borrillo, L., Guo, A. B. Y., Serrano-Wu, M. H., Lee, K. K., Hubbard, B., Johnson, J. W., Gaulin, J. L., Magolan, J., Hung, D. T., and Brown, E. D. (2021) Chemical Screen for Vancomycin Antagonism Uncovers Probes of the Gram-Negative Outer Membrane, ACS Chem Biol 16, 929-942.

7. Vaara, M. (1992) Agents that increase the permeability of the outer membrane, Microbiol Rev 56, 395-411.

8. Stokes, J. M., MacNair, C. R., llyas, B., French, S., Cote, J. P., Bouwman, C., Farha, M. A., Sieron, A. O., Whitfield, C., Coombes, B. K., and Brown, E. D. (2017) 
Pentamidine sensitizes Gram-negative pathogens to antibiotics and overcomes acquired colistin resistance, Nat Microbiol 2, 17028.

9. Hill, M. A., Lam, A. K., Reed, P., Harney, M. C., Wilson, B. A., Moen, E. L., Wright, S. N., Pinho, M. G., and Rice, C. V. (2019) BPEI-Induced Delocalization of PBP4 Potentiates beta-Lactams against MRSA, Biochemistry 58, 3813-3822.

10. Lam, A. K., Panlilio, H., Pusavat, J., Wouters, C. L., Moen, E. L., Brennan, R. E., and Rice, C. V. (2020) Expanding the Spectrum of Antibiotics Capable of Killing Multidrug-Resistant Staphylococcus aureus and Pseudomonas aeruginosa, ChemMedChem 15, 1421-1428.

11. Ferraro, N. J., and Pires, M. M. (2021) Systematic Assessment of Accessibility to the Surface of Staphylococcus aureus, ACS Chem Biol Under review.

12. Lambert, P. A. (2002) Cellular impermeability and uptake of biocides and antibiotics in Gram-positive bacteria and mycobacteria, J Appl Microbiol 92 Suppl, 46S-54S.

13. Vollmer, W., Blanot, D., and de Pedro, M. A. (2008) Peptidoglycan structure and architecture, FEMS Microbiol Rev 32, 149-167.

14. Maresso, A. W., and Schneewind, O. (2008) Sortase as a target of anti-infective therapy, Pharmacol Rev 60, 128-141.

15. Nelson, J. W., Chamessian, A. G., McEnaney, P. J., Murelli, R. P., Kazmierczak, B. I., and Spiegel, D. A. (2010) A biosynthetic strategy for re-engineering the Staphylococcus aureus cell wall with non-native small molecules, ACS Chem Biol 5, 1147-1155. 
16. Weidenmaier, C., and Peschel, A. (2008) Teichoic acids and related cell-wall glycopolymers in Gram-positive physiology and host interactions, Nat Rev Microbiol 6, 276-287.

17. Vaz, F., Kounatidis, I., Covas, G., Parton, R. M., Harkiolaki, M., Davis, I., Filipe, S. R., and Ligoxygakis, P. (2019) Accessibility to Peptidoglycan Is Important for the Recognition of Gram-Positive Bacteria in Drosophila, Cell Rep 27, 2480-2492 e2486.

18. Weidenmaier, C., Kokai-Kun, J. F., Kristian, S. A., Chanturiya, T., Kalbacher, H., Gross, M., Nicholson, G., Neumeister, B., Mond, J. J., and Peschel, A. (2004) Role of teichoic acids in Staphylococcus aureus nasal colonization, a major risk factor in nosocomial infections, Nat Med 10, 243-245.

19. Du, X., Larsen, J., Li, M., Walter, A., Slavetinsky, C., Both, A., Sanchez Carballo, P. M., Stegger, M., Lehmann, E., Liu, Y., Liu, J., Slavetinsky, J., Duda, K. A., Krismer, B., Heilbronner, S., Weidenmaier, C., Mayer, C., Rohde, H., Winstel, V., and Peschel, A. (2021) Staphylococcus epidermidis clones express Staphylococcus aureus-type wall teichoic acid to shift from a commensal to pathogen lifestyle, Nat Microbiol 6, 757-768.

20. Royet, J., and Dziarski, R. (2007) Peptidoglycan recognition proteins: pleiotropic sensors and effectors of antimicrobial defences, Nat Rev Microbiol 5, 264-277.

21. Wolf, A. J., and Underhill, D. M. (2018) Peptidoglycan recognition by the innate immune system, Nat Rev Immunol 18, 243-254.

22. Foster, T. J. (2005) Immune evasion by staphylococci, Nat Rev Microbiol 3, 948958. 
23. Lowy, F. D. (2003) Antimicrobial resistance: the example of Staphylococcus aureus, J Clin Invest 111, 1265-1273.

24. Feigman, M. S., Kim, S., Pidgeon, S. E., Yu, Y., Ongwae, G. M., Patel, D. S., Regen, S., Im, W., and Pires, M. M. (2018) Synthetic Immunotherapeutics against Gram-negative Pathogens, Cell Chem Biol 25, 1185-1194 e1185.

25. Fura, J. M., Pidgeon, S. E., Birabaharan, M., and Pires, M. M. (2016) DipeptideBased Metabolic Labeling of Bacterial Cells for Endogenous Antibody Recruitment, ACS Infect Dis 2, 302-309.

26. Fura, J. M., and Pires, M. M. (2015) D-amino carboxamide-based recruitment of dinitrophenol antibodies to bacterial surfaces via peptidoglycan remodeling, Biopolymers 104, 351-359.

27. Fura, J. M., Sabulski, M. J., and Pires, M. M. (2014) D-amino acid mediated recruitment of endogenous antibodies to bacterial surfaces, ACS Chem Biol 9, 1480-1489.

28. Fura, J. M., Sarkar, S., Pidgeon, S. E., and Pires, M. M. (2017) Combatting Bacterial Pathogens with Immunomodulation and Infection Tolerance Strategies, Curr Top Med Chem 17, 290-304.

29. Sabulski, M. J., Pidgeon, S. E., and Pires, M. M. (2017) Immuno-targeting of Staphylococcus aureus via surface remodeling complexes, Chem Sci 8, 68046809.

30. Baskin, J. M., Prescher, J. A., Laughlin, S. T., Agard, N. J., Chang, P. V., Miller, I. A., Lo, A., Codelli, J. A., and Bertozzi, C. R. (2007) Copper-free click chemistry for dynamic in vivo imaging, Proc Natl Acad Sci U S A 104, 16793-16797. 
31. Jewett, J. C., Sletten, E. M., and Bertozzi, C. R. (2010) Rapid Cu-free click chemistry with readily synthesized biarylazacyclooctynones, J Am Chem Soc $132,3688-3690$.

32. Apostolos, A. J., Pidgeon, S. E., and Pires, M. M. (2020) Remodeling of Crossbridges Controls Peptidoglycan Crosslinking Levels in Bacterial Cell Walls, ACS Chem Biol.

33. Pidgeon, S. E., Apostolos, A. J., Nelson, J. M., Shaku, M., Rimal, B., Islam, M. N., Crick, D. C., Kim, S. J., Pavelka, M. S., Kana, B. D., and Pires, M. M. (2019) L,DTranspeptidase Specific Probe Reveals Spatial Activity of Peptidoglycan CrossLinking, ACS Chem Biol 14, 2185-2196.

34. Welsh, M. A., Taguchi, A., Schaefer, K., Van Tyne, D., Lebreton, F., Gilmore, M. S., Kahne, D., and Walker, S. (2017) Identification of a Functionally Unique Family of Penicillin-Binding Proteins, J Am Chem Soc 139, 17727-17730.

35. Gautam, S., Kim, T., Shoda, T., Sen, S., Deep, D., Luthra, R., Ferreira, M. T., Pinho, M. G., and Spiegel, D. A. (2015) An Activity-Based Probe for Studying Crosslinking in Live Bacteria, Angew Chem Int Ed Engl 54, 10492-10496.

36. Gautam, S., Kim, T., and Spiegel, D. A. (2015) Chemical probes reveal an extraseptal mode of cross-linking in Staphylococcus aureus, J Am Chem Soc $137,7441-7447$.

37. Ngadjeua, F., Braud, E., Saidjalolov, S., Iannazzo, L., Schnappinger, D., Ehrt, S., Hugonnet, J. E., Mengin-Lecreulx, D., Patin, D., Etheve-Quelquejeu, M., Fonvielle, M., and Arthur, M. (2018) Critical Impact of Peptidoglycan Precursor 
Amidation on the Activity of I,d-Transpeptidases from Enterococcus faecium and Mycobacterium tuberculosis, Chemistry.

38. D'Elia, M. A., Millar, K. E., Beveridge, T. J., and Brown, E. D. (2006) Wall teichoic acid polymers are dispensable for cell viability in Bacillus subtilis, J Bacteriol 188, 8313-8316.

39. Swoboda, J. G., Meredith, T. C., Campbell, J., Brown, S., Suzuki, T., Bollenbach, T., Malhowski, A. J., Kishony, R., Gilmore, M. S., and Walker, S. (2009) Discovery of a small molecule that blocks wall teichoic acid biosynthesis in Staphylococcus aureus, ACS Chem Biol 4, 875-883.

40. Gautam, S., Kim, T., Lester, E., Deep, D., and Spiegel, D. A. (2016) Wall teichoic acids prevent antibody binding to epitopes within the cell wall of Staphylococcus aureus, ACS Chem Biol 11, 25-30.

41. Eugster, M. R., and Loessner, M. J. (2012) Wall teichoic acids restrict access of bacteriophage endolysin Ply118, Ply511, and PlyP40 cell wall binding domains to the Listeria monocytogenes peptidoglycan, J Bacteriol 194, 6498-6506.

42. Novick, R. P., Projan, S. J., Kornblum, J., Ross, H. F., Ji, G., Kreiswirth, B., Vandenesch, F., and Moghazeh, S. (1995) The agr P2 operon: an autocatalytic sensory transduction system in Staphylococcus aureus, Mol Gen Genet 248, 446-458.

43. Cosgriff, C. J., White, C. R., Teoh, W. P., Grayczyk, J. P., and Alonzo, F., 3rd. (2019) Control of Staphylococcus aureus Quorum Sensing by a MembraneEmbedded Peptidase, Infect Immun 87. 
44. Singh, V. K., Sirobhushanam, S., Ring, R. P., Singh, S., Gatto, C., and Wilkinson, B. J. (2018) Roles of pyruvate dehydrogenase and branched-chain alpha-keto acid dehydrogenase in branched-chain membrane fatty acid levels and associated functions in Staphylococcus aureus, J Med Microbiol 67, 570-578.

45. Grundling, A., and Schneewind, O. (2006) Cross-linked peptidoglycan mediates lysostaphin binding to the cell wall envelope of Staphylococcus aureus, $J$ Bacteriol 188, 2463-2472. 